ARTICLE

\title{
Structural insight into the molecular mechanism of p53-mediated mitochondrial apoptosis
}

Hudie Wei ${ }^{1}$, Lingzhi Qu${ }^{1}$, Shuyan Dai ${ }^{1}$, Yun $\mathrm{Li}^{1}$, Haolan Wang${ }^{1}$, Yilu Feng ${ }^{1}$, Xiaojuan Chen ${ }^{1}$, Longying Jiang ${ }^{1}$, Ming Guo ${ }^{1}$, Jun $\mathrm{Li}^{1}$, Zhuchu Chen ${ }^{1}$, Lin Chen ${ }^{2}$, Ye Zhang ${ }^{1 凶} \&$ Yongheng Chen (1) ${ }^{1,3 凶}$

The tumor suppressor p53 is mutated in approximately half of all human cancers. p53 can induce apoptosis through mitochondrial membrane permeabilization by interacting with and antagonizing the anti-apoptotic proteins $B C L-x L$ and $B C L-2$. However, the mechanisms by which p53 induces mitochondrial apoptosis remain elusive. Here, we report a $2.5 \AA$ crystal structure of human $\mathrm{p} 53 / \mathrm{BCL}-\mathrm{xL}$ complex. In this structure, two p53 molecules interact as a homodimer, and bind one BCL-XL molecule to form a ternary complex with a 2:1 stoichiometry. Mutations at the p53 dimer interface or p53/BCL-xL interface disrupt p53/BCL-xL interaction and p53-mediated apoptosis. Overall, our current findings of the bona fide structure of $\mathrm{p} 53 / \mathrm{BCL}-\mathrm{xL}$ complex reveal the molecular basis of the interaction between $\mathrm{p} 53$ and $\mathrm{BCL}-\mathrm{XL}$, and provide insight into $\mathrm{p} 53$-mediated mitochondrial apoptosis.

\footnotetext{
${ }^{1}$ Department of Oncology, NHC Key Laboratory of Cancer Proteomics, Laboratory of Structural Biology, Xiangya Hospital, Central South University, Changsha, Hunan, China. ${ }^{2}$ Molecular and Computational Biology Program, Department of Biological Sciences and Department of Chemistry, University of Southern California, Los Angeles, CA, USA. ${ }^{3}$ National Clinical Research Center for Geriatric Disorders, Xiangya Hospital, Central South University, Changsha, Hunan, China. ${ }^{凶}$ email: yezhang90@csu.edu.cn; yonghenc@163.com
} 
T he tumor suppressor p53 plays central roles in regulating cell-cycle arrest, DNA repair, and apoptosis in the response to cellular stress ${ }^{1}$. Mutations of p53 have been reported in about half of all human cancers, highlighting its essential role in cancer suppression ${ }^{2,3}$. Full-length p53 protein contains a N-terminal transactivation domain (TAD), a prolinerich region, a DNA-binding domain (DBD), a tetramerization domain, and a C-terminal regulatory domain ${ }^{3}$. Human p53 is active as a homotetramer. In addition to assembly through the tetramerization domain, p53-DBD forms a homotetramer with a dimer-of-dimers topology upon DNA binding ${ }^{4-6}$.

The induction of apoptosis is crucial for the tumor-suppressive activity of $\mathrm{p} 53^{7,8}$. As a nuclear transcription factor, $\mathrm{p} 53$ regulates many genes involved in cell apoptosis. Notably, p53 has been reported to migrate to the mitochondria, and mediates the mitochondrial apoptosis pathway (also called the intrinsic pathway) primarily through direct protein-protein interactions with $B$ cell lymphoma-2 (BCL-2) family proteins ${ }^{9-11}$. The BCL-2 family proteins, which include both pro-apoptotic and anti-apoptotic members, control mitochondrial outer-membrane permeabilization (MOMP) and consequent activation of the caspase cascade ${ }^{12}$.

BCL-2 and BCL-xL are anti-apoptotic members of BCL-2 family, which inhibit apoptosis by directly engaging the effector proteins $\mathrm{BAK}$ and $\mathrm{BAX}$, or sequestering pro-apoptotic $\mathrm{BH} 3$-only members ${ }^{13}$. Dysregulation of BCL-2 and BCL-xL is commonly associated with tumorigenesis and chemotherapy resistance ${ }^{14,15}$. P53 has been reported to directly interact with and antagonize BCL-2 and BCL-xL, thereby leading to effector activation and ultimately apoptosis ${ }^{11}$. The DNA-binding domain of p53 mediates its interactions with BCL-xL and BCL-2 at a surface outside the hydrophobic groove for $\mathrm{BH} 3$ peptide binding ${ }^{16-20}$. The tetramerization of $\mathrm{p} 53$ enhances the binding to $\mathrm{BCL}-\mathrm{xL}^{17}$. A structural model of p53/BCL-xL complex has been previously proposed using HADDOCK docking method ${ }^{21}$ based on 1:1 stoichiometry ${ }^{17}$.

In this work, we determine a $2.5 \AA$ crystal structure of human p53/BCL-xL complex. Unlike the structural model based on 1:1 stoichiometry, our structure and biochemical studies show that two p53 molecules and one BCL-xL molecule form a ternary complex with a 2:1 stoichiometry. Our studies also show that mutations at the p53 dimer interface or p53/BCL-xL interface disrupt p53/BCL-xL interaction and p53-mediated apoptosis. Overall, our bona fide structure of p53/BCL-xL complex reveals the molecular basis of the interaction between p53 and BCL-xL, and provides insight into p53-mediated mitochondrial apoptosis.

\section{Results}

Crystal structure of the p53/BCL-xL complex. To investigate the molecular basis of the interaction between $\mathrm{p} 53$ and BCL-xL, we proceeded to determine the crystal structure of p53/BCL-xL complex. In order to increase the probability of crystallization, two protein constructs were tested based on previous structural studies: p53-DBD (amino acids 92-2924), and a BCL-xL construct $^{22}$ lacking the C-terminal transmembrane region and the disordered $\alpha 1-\alpha 2$ loop which does not participate in p53 binding ${ }^{16-19,23}$ (Supplementary Fig. 1a-b). Initially we tried to crystallize this complex by mixing $\mathrm{p} 53-\mathrm{DBD}$ and $\mathrm{BCL}-\mathrm{xL}$ proteins, but it did not work, probably due to the weak and transient interaction between p53 and BCL-xL. To keep the two proteins in close proximity, we fused the BCL-xL via a glycine-rich linker ${ }^{24}$ to p53-DBD which was reported to mediate interactions with BCL-xL ${ }^{16-19}$ (Supplementary Fig. 1c). Using this strategy, we determined the crystal structure of the fusion protein at a $2.5 \AA$ resolution in the P $2_{1}$ space group (Supplementary Table 1). In the structure, an asymmetric unit comprises two BCL-xL and two
p53-DBD molecules (Fig. 1a). The two p53 molecules (molecules $\mathrm{A}$ and B) contact each other and form a groove. One BCL-xL molecule (molecule C) inserts deep into the groove, and interacts with both p53 molecules (Fig. 1b). However, the other BCL-xL molecule (molecule D) sits outside the groove, and interacts with the p53 molecules to a much lesser extent (Fig. 1c). Based on our structural observation, we speculated that the interaction between the p53 dimer and molecule D could be formed due to crystal packing (Supplementary Fig. 2).

To test our hypothesis, we first carried out crosslinking analyse $^{25}$ to examine how p53 tetramer binds BCL-xL in solution. We used a p53 construct containing both DBD and tetramerization domain (termed p53-DT ${ }^{17}$, amino acids 92-360). The gel-filtration chromatography indicated that p53-DT existed as tetramer in solution, while p53-DBD existed as monomer in solution (Supplementary Fig. 3a). The p53-DT protein was incubated with $1 \%$ glutaraldehyde in the presence or absence of BCL-xL. Then the mixtures were subjected to SDS/PAGE and silver staining. p53-DT protein (about $30 \mathrm{kDa}$ ) preferentially migrated at a band of about $120 \mathrm{kDa}$ (Fig. 1d, lane 3), indicating a p53-DT tetramer band. In the presence of BCL-xL (about 20 $\mathrm{kDa}$ ), a complex band of about $160 \mathrm{kDa}$ molecular weight was observed (Fig. 1d, lane 2), suggesting that p53-DT forms a 4:2 complex with BCL-xL. We also tried p53-DBD and BCL-xL in the crosslinking experiment. In the presence of BCL-xL, a complex band of about $70 \mathrm{kDa}$ was observed, suggesting that $\mathrm{p} 53-\mathrm{DBD}$ formed a 2:1 complex with BCL-xL (Supplementary Fig. 3b).

In addition, GST-pull down assays were performed to examine interactions of p53-DBD and p53-DT with BCL-xL (Supplementary Fig. 4). Both p53-DBD and p53-DT were pulled down by GST-BCL-xL, with p53-DT showing stronger binding. A quantitative measurement was performed using isothermal titration calorimetry (ITC) (Fig. 1e, Supplementary Fig. 5). p53DT bound BCL-xL with a $\mathrm{KD}$ value of $4.1 \mu \mathrm{M}$ in a ratio of $2: 1$ (Fig. 1e), while p53-DBD interacted with BCL-xL with a Kd value of $37.4 \mu \mathrm{M}$ (Supplementary Fig. 5). These results are consistent with previous findings that the tetramerization of p53 enhances the binding to $\mathrm{BCL}-\mathrm{xL}^{17}$, and support our hypothesis that $\mathrm{p} 53$ binds BCL-xL with a stoichiometry of 2:1.

The p53-DBD dimer interface. The structure of BCL-xL/p53 complex shows that the two p53-DBD molecules dimerize through an interface of approximately $900 \AA^{2}$. It mainly involves residues in the N-terminal arm of p53-DBD and the loop linking $\beta 9$ and $\beta 10$ of molecule $\mathrm{p} 53-\mathrm{A}$, and residues in loop 2, helix 1 and loop 3 of molecule p53-B (Fig. 1). Residue His178 of p53-B forms a hydrogen bond and a salt bridge with residues Tyr107 and Asp259 of p53-A, respectively (Fig. 2a, Supplementary Fig. 6). His179 from the $\mathrm{Zn}^{2+}$ coordination site of $\mathrm{p} 53-\mathrm{B}$ also forms a hydrogen bond with residue Tyr107 of p53-A. Moreover, residues Asn239 and Ser241 of p53-B interact through hydrogen bonds with the backbone of Ser106 and Gly105 of p53-A, respectively. Met243 of p53-B establishes hydrophobic contacts with Tyr103, Leu264 and Gly265 of p53-A. These interactions and many other van der Waals interactions help stabilize the p53 dimer (Fig. 2b).

We then compared the p53 dimer interface with previously reported $\mathrm{p} 53$ structures. The $\mathrm{p} 53$ dimer interface in our structure (A-B dimer) is highly similar to a p53 dimer (B-D dimer) in the structure of human p53-DBD in the absence of $\mathrm{DNA}^{26}$. The overall structures of the two dimers are largely overlapped with a RMSD value of $0.56 \AA$ based on Ca atoms (Supplementary Fig. 7). The interfacial residues in dimer interfaces form similar hydrogen bond interactions. Little change was identified in this interface upon BCL-xL binding. Such p53 dimers appear to be an intrinsic property of $\mathrm{p} 53-\mathrm{DBD}^{26,27}$ that allows for further interaction with 


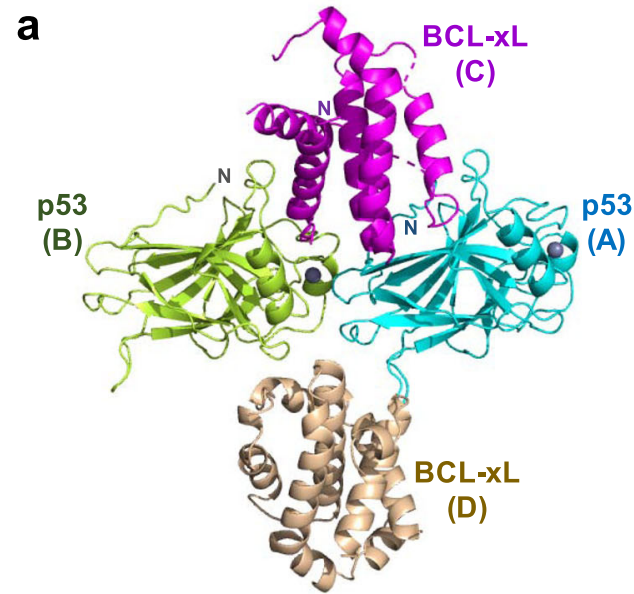

b

C

d

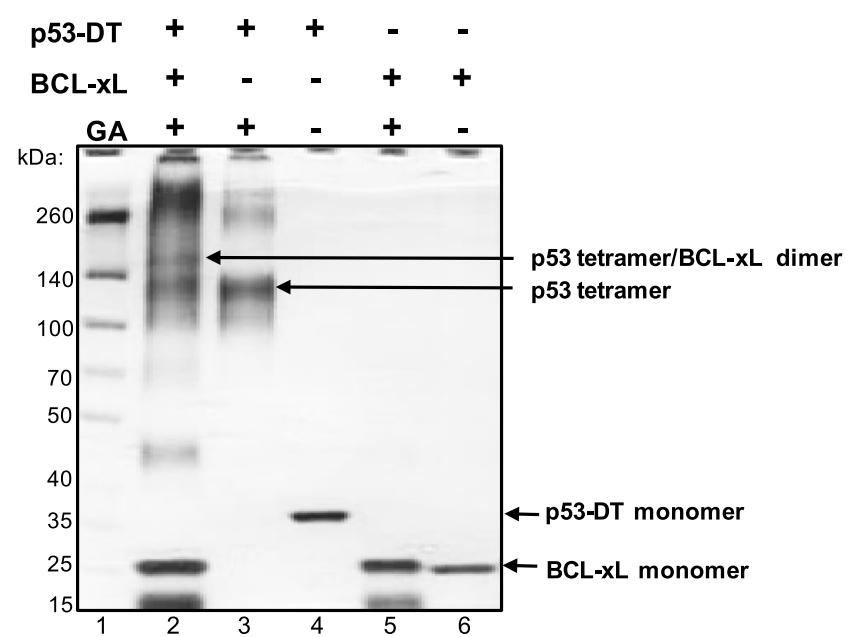

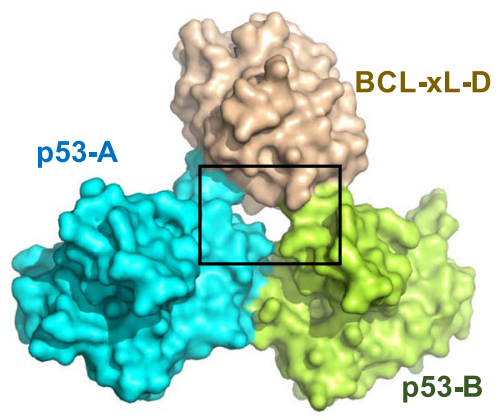

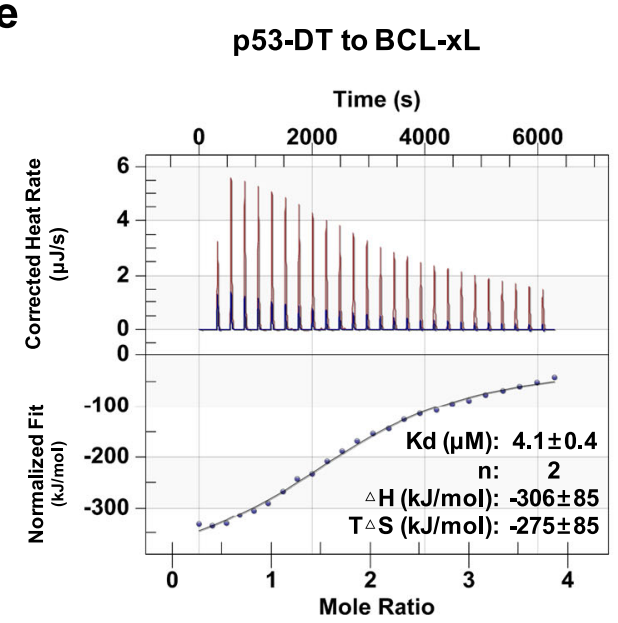

Fig. 1 The complex formation of $\mathbf{p 5 3}$ and BCL-xL. a Overall structure of p53/BCL-xL complex. An asymmetric unit of p53/BCL-xL complex comprises two p53-DBD molecules (colored cyan and limon) and two BCL-xL molecules (colored magenta and wheat). $\mathbf{b} A$ surface model of the ternary complex composed of the $\mathrm{p} 53$ dimer and $\mathrm{BCL}-\mathrm{xL}-\mathrm{C}$. BCL-xL-C molecule inserted deep into the groove formed by the p53 dimer. c $\mathrm{A}$ surface model of the ternary complex composed of the $\mathrm{p} 53$ dimer and BCL-xL-D. BCL-xL-D molecule sits outside the groove formed by the p53 dimer. d The complex formation of $p 53-$ DT and BCL-xL analyzed by protein crosslinking and SDS-PAGE. Purified p53-DT, BCL-xL or their mixture were incubated in the presence or absence of $0.1 \%$ glutaraldehyde (GA) as indicated. Products of the crosslinking reactions were analyzed by SDS-PAGE followed by silver staining. The experiments were repeated independently more than 3 times with similar results and a representative experiment is shown. e The binding affinity between p53-DT and $\mathrm{BCL}-\mathrm{xL}$ was measured using isothermal titration calorimetry (ITC) assays. The binding parameters indicate average of three independent titrations \pm SEM Source data are provided as a Source Data file.

BCL resulting in a $2: 1$ complex. In addition, we find that this interface is different from the previously reported dimer interface and dimer-dimer interface in the p53-DBD/DNA complex ${ }^{4,28}$ (Supplementary Fig. 8).

The p53/BCL-xL interface. In our structure, the p53-DBD dimer covers an interface of approximately $1600 \AA^{2}$ with BCL-xL-C. This BCL-xL binding interface comprises residues from the $\mathrm{N}$ terminal loop, $\beta 1$ and $\beta 10$ of $\mathrm{p} 53-\mathrm{A}$, and from the helix $2, \beta 10$ and loop3 of p53-B (Fig. 1). The relevant residues on the BCL-xL surface include the C-terminal residues of $\alpha 1$ and $\alpha 5$, the $\alpha 5-\alpha 6$ loop and the $\alpha 3-\alpha 4$ loop. Tyr22 and Asp156 of BCL-xL contact with Arg248 of p53-B through a hydrogen bond and a salt bridge contact, respectively (Fig. 2c, d, Supplementary Fig. 9). The backbone oxygen atom of the BCL-xL residue Asp156 also contributes hydrogen bonds and water-mediated interactions with residues Gln104 and Asn268 of p53-A. In addition, His113 of BCL-xL forms a hydrogen bond with $\mathrm{Gln} 100$ of p53-A. The side chain of p53-B residue Arg273 is stabilized by hydrogen bond interactions with BCL-xL backbone groups (Fig. 2c, d, Supplementary Fig. 9).

BCL-xL binds $\mathrm{BH} 3$ peptide via a hydrophobic binding groove $^{29}$, we then compared p53-bound BCL-xL in our structure with BID-BH3 bound BCL-xL (PDB: 4QVE) ${ }^{30}$. As shown in Supplementary Fig. 10, p53 binds BCL-xL at a surface outside the hydrophobic groove. Little conformational changes were observed in the hydrophobic groove due to the p53 binding (Supplementary Fig. 10).

Previous structural studies show that BCL-xL forms a dimer through three-dimensional domain swapping (3DDS) by swapping helices $a 6-\alpha 8$ between two monomers ${ }^{31,32}$. We superposed our structure with domain-swapped BCL-xL structure (PDB: $4 \mathrm{PPI})^{32}$, and found that p53 would clash with the domainswapped BCL-xL homodimer, suggesting that p53 may inhibit BCL-xL homodimerization (Supplementary Fig. 11).

Comparison with previously proposed structural model. We then compared our structure with the previously proposed structural model ${ }^{17}$. As shown in Fig. 3, the p53-interaction 
a

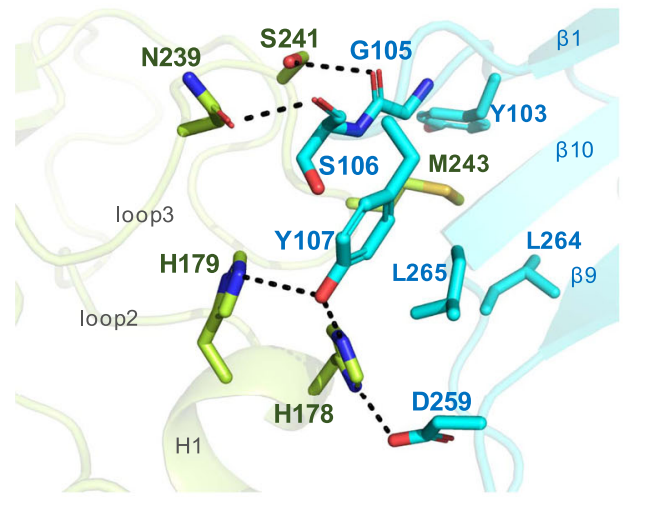

b

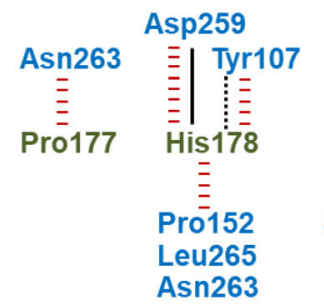

C

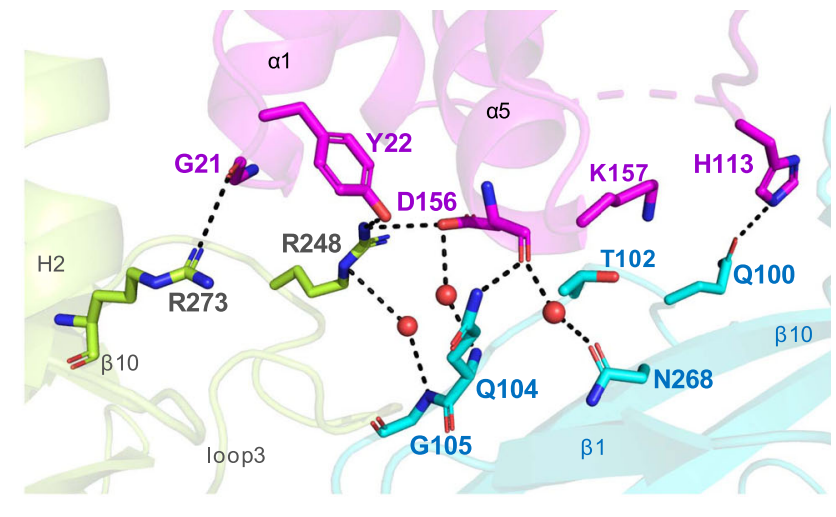

Leu265

Leu264

Tyr103

$\equiv$

Mèt243
p53-A residues p53-B residues $\quad B C L-x L$ residues

— Salt bridge

......... Hydrogen bond involving side-chain groups

- - - Hydrogen bond involving backbone groups

I 1 । । Non-bonded contact

- Water molecular

d
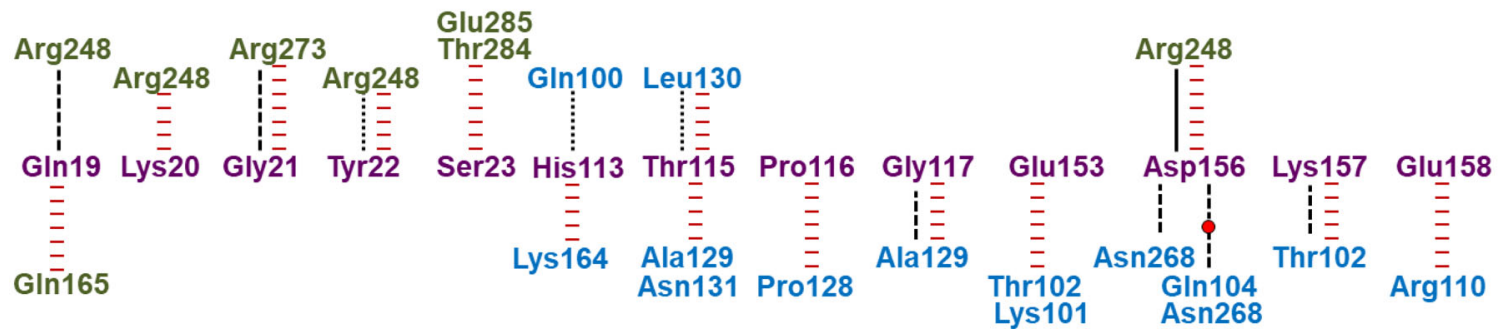

Fig. 2 Detailed contacts in the p53/BCL-xL complex. a Key interactions at the p53 dimer interface. Involved residues are shown as sticks. Polar contacts are shown as black dotted lines. Red spheres represent water molecules. b Schematic diagram of the detailed contacts at the p53 dimer interface. c Key interactions between p53 dimer and BCL-xL. d Schematic diagram of the detailed interactions at the binding interface.

surface on BCL-xL is in good agreement with the previous model. Most of the residues that were predicted to interact with p53 in the structural model indeed binds p53 in our structure (Fig. 3b). It is worth noting that a region of BCL-xL (amino acids 99-111) has poor electron density in BCL-xL-C, although this region has clear electron density in BCL-xL-D, suggesting that this region becomes flexible upon p53 binding. Part of this region (amino acids 102-105) was predicted to interact with $\mathrm{p} 53$, however, these residues do not directly interact with p53 in our structure (Fig. 3b). On the other hand, the BCL-xL interaction surface on p53 is very different between our structure and the previous model (Fig. 3). In our structure, two p53-DBD molecules dimerize to interact with one molecule of $\mathrm{BCL}-\mathrm{xL}$; while in the previous model, one p53-DBD molecule interacts with one BCL-xL molecule. Among all the residues that were predicted to interact with BCL-xL in the previous model, only Arg248 interacts with BCL-xL directly in our structure (Fig. 3c, right panel, highlighted in orange). Five residues that are projected to bind BCL-xL in the previous model (Fig. 3c, right panel, highlighted in red), are involved in p53 dimerization in our structure.

Mutational analysis of the p53/BCL-xL interactions. We then tested the relevance of the observed binding interfaces through structure-guided mutagenesis of both p53 and BCL-xL. On the p53 side, Y107A and H178A mutants were generated at the p53 dimer interface, and R248A, R273A and R273C mutants were generated at the $\mathrm{p} 53 / \mathrm{BCL}-\mathrm{xL}$ interface. On the $\mathrm{BCL}-\mathrm{xL}$ side, Y22A and D156R were generated at the p53/BCL-xL interface. It is worth noting that Arg248 and Arg273 residues of p53 are also in DNA contact positions, and are well-known sites of hotspot mutations that reduce p53 activity (or even cause gain-offunction depending on what mutation occurs) ${ }^{3}$.

The effect of these mutations on the p53/BCL-xL interaction was assessed using co-immunoprecipitation assays. All designed p53 mutants expressed in p53-null H1299 cells showed decreased co-immunoprecipitation with endogenous BCL-xL (Fig. 4a). In agreement with our structural observation that p53 dimerization is important for $\mathrm{p} 53 / \mathrm{BCL}-\mathrm{xL}$ interaction, although Y107A and H178A mutations do not locate at the p53/BCL-xL interfaces, they disrupt the interaction between p53 and BCL-xL. On the BCL-xL side, over-expressed BCL-xL mutants Y22A and D156R showed decreased co-immunoprecipitation with wild-type p53 (Fig. 4b). The effect of the designed mutations on the p53/BCL-xL interaction was further assessed by isothermal titration calorimetry. The mutants Y107A, H178A, R248A and R273A showed 5-12-fold decreased binding affinity to BCL-xL compared with wild-type p53 (Fig. 4c, Supplementary Fig. 12). Taking together, these structure-guided mutagenesis assays supported the interfaces defined by the BCL-xL/p53 complex structure. 
a

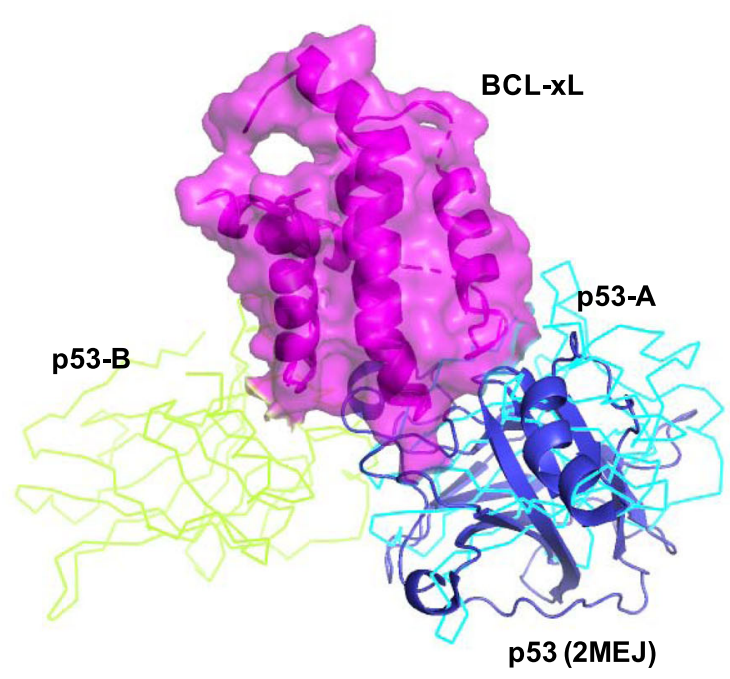

b

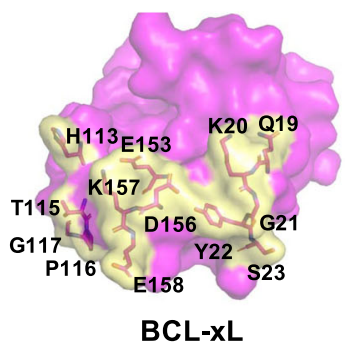

C

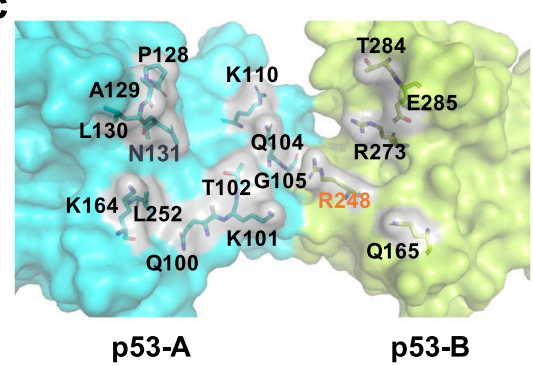

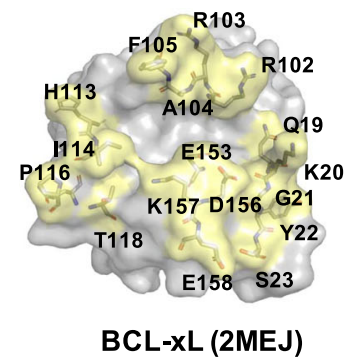

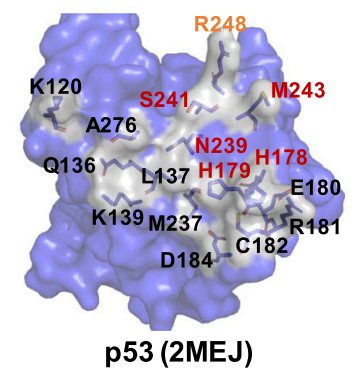

Fig. 3 Comparison with previously proposed structural model. a Our $B C L-x L / p 53$ structure is superimposed on the previous structural model (PDB: $2 \mathrm{MEJ}$ ) using $B C L-x L-C$ as the reference. A surface model of $B C L-x L$ is shown in magenta, $p 53-A$ is shown as cyan ribbon, $p 53-B$ is shown as limon ribbon, the p53 molecule from the previous model is shown as blue cartoon. $\mathbf{b}$ A detailed comparison of the interaction surfaces on $B C L-x L$. The $B C L-x L$ in our structure is shown as magenta surface. The BCL-xL in the previous structural model is shown as gray surface. The interface is shown in yellow, with all the interaction residues labeled. c. A detailed comparison of the interaction surfaces on p53-DBD. The p53-DBD dimer in our structure is shown as cyan and limon surface. The p53-DBD in the previous model is shown as blue surface. The interface is shown in gray, with all the interaction residues labeled. Arg248 (labeled in orange) interacts with BCL-xL in both structures. Residues labeled in red are projected to bind BCL-xL in the previous model, while they are involved in $\mathrm{p} 53-\mathrm{DBD}$ dimerization in our structure.

a

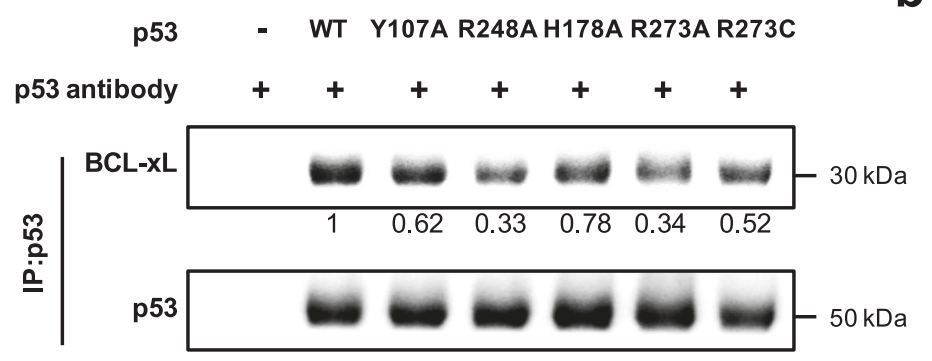

b

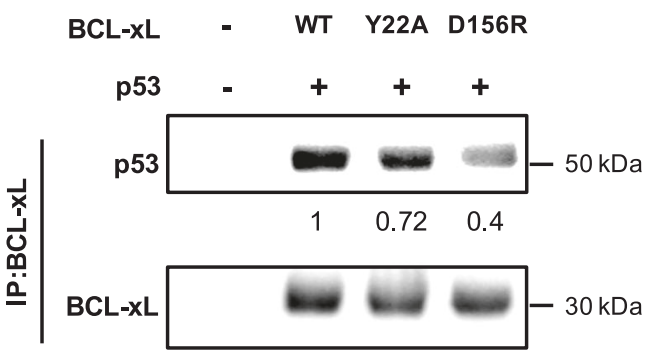

C

\begin{tabular}{cccccc}
\hline p53-DT & WT & Y107A & H178A & R248A & R273A \\
\hline KD $(\mu \mathrm{M})$ & $4.1 \pm 0.4$ & $28.5 \pm 4.4$ & $49.4 \pm 6.5$ & $48.3 \pm 0.4$ & $20.8 \pm 3.6$ \\
Mutant/WT & 1 & 6.9 & 12.1 & 11.7 & 5.1 \\
\hline
\end{tabular}

Fig. 4 Mutations of interaction residues of p53 and BCL-xL disrupt their interaction. a Wild-type or mutant p53 plasmid was transiently transfected into p53-null H1299 cells. Cell lysates were immunoprecipitated using anti-p53 antibody, and subjected to western blotting as indicated. b Wild-type or mutant BCL-xL plasmid (flag-tagged) was co-transfected with wild-type p53 plasmid into H1299 cells. Cell lysates were immunoprecipitated using anti-flag antibody, and subjected to western blotting as indicated. The results of three independent replicates were similar, with one representative experiment being shown. Semi-quantitation using band densitometry was performed and the relative binding compared to wild type was show below. $\mathbf{c}$ ITC assay demonstrated affinity of wild-type or mutant p53-DT to $B C L-x L$. The KD values indicate average of three independent titrations \pm SEM. The relative binding affinity (Mutant/WT) is indicated. Source data are provided as a Source Data file.

Interfacial mutants inhibit p53-mediated apoptosis. The inhibition of anti-apoptotic BCL-xL is a significant part of $\mathrm{p} 53$ mediated apoptosis. We then tested the effect of the designed p53 mutants on p53-mediated apoptosis. P53-null H1299 cells were transfected with wild-type or mutant p53 for 48 hours, followed by Annexin V/PI staining and flow cytometry assay. The data showed that the transfection of wild-type p53 induced approximately $26.7 \%$ of apoptotic cells, whereas H178A, R248A and R273A mutants led to about $9.4 \%, 8.9 \%$ and $12 \%$ of apoptotic cells, respectively (Fig. 5a, b, Supplementary Fig. 13). In addition, 
a
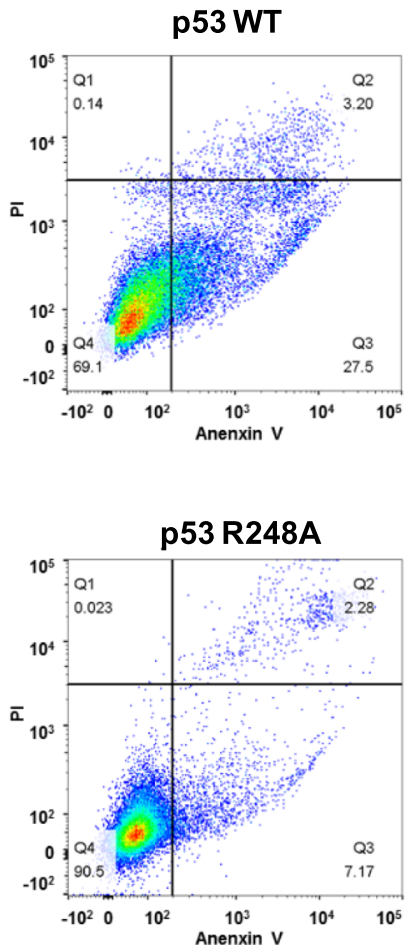

p53 H178A

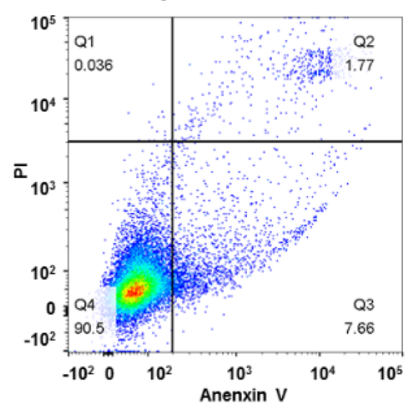

p53 R273C

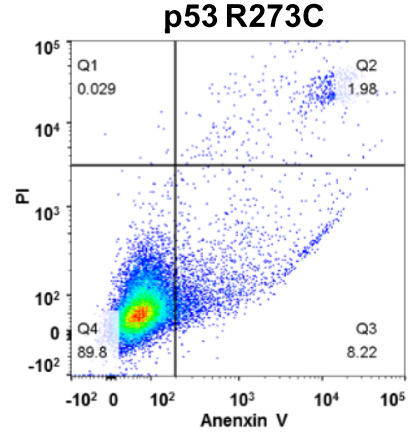

b

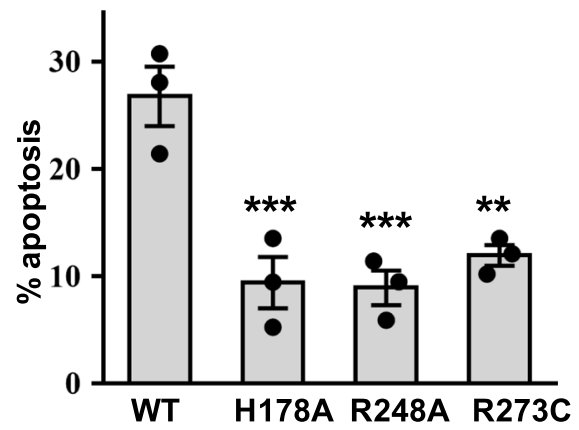

C
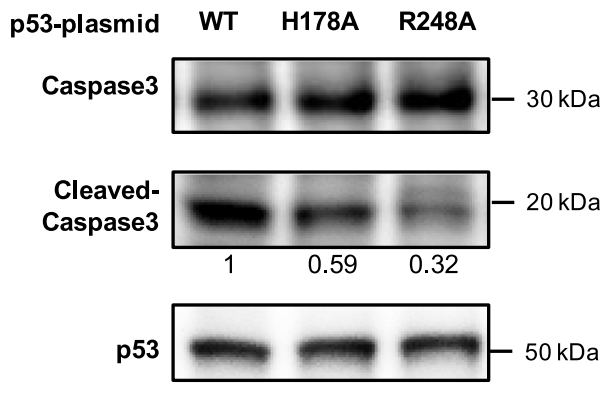

Fig. 5 Interfacial mutations inhibit p53-mediated apoptosis. a H1299 cells were transiently transfected with wild-type or mutant p53 plasmids. The apoptosis rate of $\mathrm{H} 1299$ cells was analyzed by flow cytometry using Annexin V/PI staining. One representative experiment was shown, as three independent replicates were similar. $\mathbf{b}$ Ratio of apoptotic cells in the flow cytometry assay. Data are presented as dot, $n=3$ independent replicates. Error bars indicate mean \pm SEM. $P$-values were determined by one way ANOVA followed by Dunnett's multiple comparisons test using wild-type group as a control, ${ }^{\star \star} P<0.01,{ }^{\star \star \star} P<0.001$. $P=0.0009$ for H178A, $P=0.0007$ for H178A, $P=0.0024$ for R273C. Source data are provided as a Source Data file. c Caspase 3 activation was detected by western blotting using anti-caspase 3 antibody. Semi-quantitation using band densitometry was performed and the relative binding compared to wild type was show below.

western blotting analyses showed less cleavage of caspase 3 caused by $\mathrm{H} 178 \mathrm{~A}$ and $\mathrm{R} 248 \mathrm{~A}$ mutants (Fig. 5c). These results suggested that the p53 mutants H178A, R248A and R273C disrupted the pro-apoptotic activity of p53. Noteworthy, Arg248 and Arg273 residues are in DNA contact positions, so the reduced activity of R248A and R273C could also be due to a combination of lack of DNA contact as well as disruption of p53-BCL-xL interaction.

BCL-2 likely binds p53 in a similar mechanism. The antiapoptotic member BCL-2 shares $60 \%$ sequence similarity with BCL-xL (Fig. 6a). From a sequence comparison, we observed that the p53-interacting residues on BCL-xL (Fig. 6a, labeled by red star) are also highly conserved with the corresponding residues on BCL-2. We carried out ITC assays to measure the binding affinity between p53 and BCL-2 (Supplementary Fig. 14), and found that the affinity of p53-DT protein for BCL-2 (Kd: $3.3 \mu \mathrm{M})$ was comparable to that of BCL-xL. We then superposed the BCL2 structure (PDB: $2 \mathrm{XA} 0)^{33}$ with the BCL-xL in our structure (Fig. 6b). BCL-2 accommodate well in the groove formed by p53 dimer. The interfacial residues show highly similarity (Fig. 6c). These results suggest that BCL-2 might interact with p53 in a similar manner.

\section{Discussion}

Apoptosis is a natural mechanism to remove unwanted cells from the body, and plays an essential role in organism development and tissue homeostasis ${ }^{34}$. Resistance to apoptosis by dysregulation of BCL-2 members-controlled mitochondrial pathway is a hallmark of cancers ${ }^{35}$. The tumor suppressor p53 can mediate apoptosis by transcriptional regulation of some pro-apoptotic genes, such as death receptor DR5 and BCL-2 family members PUMA, Noxa and $\mathrm{BAX}^{36}$. In addition to the transcription activity, p53 was found to induce MOMP directly through interacting with and inhibiting anti-apoptotic members (BCL-2, BCL-xL), as well as by activating pro-apoptotic members (BAK, $\mathrm{BAX})^{9-11}$, thus triggering mitochondrial apoptosis by transcription-independent process. Our structure of the p53/ BCL-xL complex elucidated the molecular mechanism of $\mathrm{p} 53 /$ $\mathrm{BCL}-\mathrm{xL}$ interactions, providing insight into the transcriptionindependent activity of p53-mediated apoptosis.

The DNA binding domain of p53 not only binds DNA, but also plays important roles in protein-protein interactions. Previous studies have shown that $\mathrm{p} 53$-DBD binds DNA as a tetramer (4:1 ratio), while it interacts with HPV E6, Large $\mathrm{T}$ antigen, $53 \mathrm{BP} 1$, and 53BP2 in a ratio of 1:1. Our structure shows that $\mathrm{p} 53$ $\mathrm{DBD}$ binds $\mathrm{BCL}-\mathrm{xL}$ in a ratio of $2: 1$, and the $\mathrm{BCL}-\mathrm{xL}-$ binding interface on $\mathrm{p} 53-\mathrm{DBD}$ is different from previous complex of p53 with HPV oncoprotein E6 ${ }^{37}$, large T antigen (LTag) of oncovirus $\mathrm{SV}_{40}{ }^{38}, 53 \mathrm{BP} 1^{39}, 53 \mathrm{BP} 2^{40}$, or with $\mathrm{DNA}^{4-6,28}$ (Supplementary Fig. 15). Interestingly, the $\mathrm{p} 53$ dimer (A-B dimer) in our structure is highly similar to a p53 dimer (B-D dimer) in a previously solved p53-DBD structure ${ }^{26}$. Such p53 dimers appear to be an intrinsic property of $\mathrm{p} 53-\mathrm{DBD}$ that allows for further interaction with BCL-xL resulting in a 2:1 complex.

The crystal structure presented here is quite different form the structural NMR model proposed previously ${ }^{17}$. The NMR model was calculated based on 1:1 stoichiometry, however, we find that p53 and BCL-xL form complex with a 2:1 stoichiometry in our crystal structure. Our structure was supported by crosslinking 
a

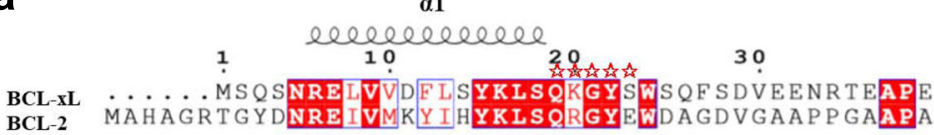

b

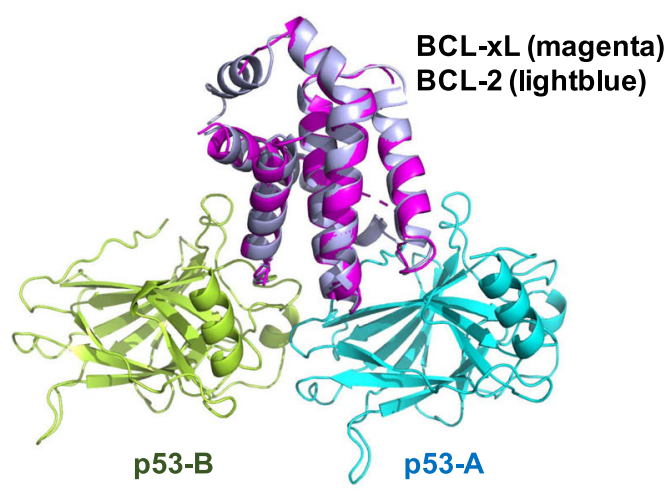

C

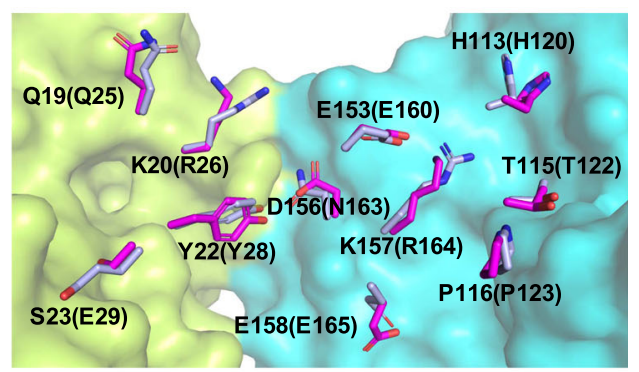

Fig. 6 BCL-2 likely binds p53 in a similar mechanism like BCL-xL. a Sequence alignment of the proteins BCL-xL and BCL-2. The sequence alignment was created using the web services ESPript 3.0. Identical and similar residues are boxed in red and white, respectively. Residues of $B C L-x L$ involved in $p 53$ binding are labeled by red star. $\mathbf{b}$ Superposition of BCL-2 structure (PDB: $2 X A 0$ ) with BCL-xL in our p53/BCL-xL complex. p53/BCL-xL complex is shown as previously. $\mathrm{BCL}-2$ is colored lightblue. $\mathbf{c}$ Interfacial residues of $\mathrm{BCL}-\mathrm{xL}$ and the corresponding residues on $\mathrm{BCL}-2$ (labeled in brackets) are shown as sticks.

and ITC analyses. Our structural observations were also supported by structure-guided mutagenesis assays. Mutations at p53/ BCL-xL interfaces, such as R248A and R273A, disrupt the interaction between $\mathrm{p} 53$ and $\mathrm{BCL}-\mathrm{xL}$. More importantly, although mutations at p53 dimer interface, such as Y107A and H178A, are not located at the p53/BCL-xL interfaces, they still disrupt the interaction, indicating that the formation of $\mathrm{p} 53$ dimer is important for the interaction between p53 and BCL-xL.

Mutation of the p53 tumor suppressor is a frequent event in all human cancers. Among all the p53 mutations, about 30\% fall within six hotspot residues (including Arg175, Gly245, Arg248, Arg249, Arg273, and Arg282) ${ }^{41}$. Two of the six hotspot residues, Arg248 and Arg273, are located at the p53/BCL-xL interfaces, and mutations at these two residues disrupt the interaction. It is worth noting that these two residues also play important roles in DNA binding and mutations at these residues result in loss of DNA binding. Therefore, mutations at these two hotspot residues could serve as 'double hit' mutants for both nuclear transcriptiondependent and mitochondria transcription-independent apoptosis. In addition, some mutations could also lead to changes in protein stability and hence reduction in the stabilization of a p53/ BCL-xL complex, or interfering with other p53 functions.

In summary, our crystal structure of the p53/BCL-xL complex shows that p53 interacts as a dimer with BCL-xL. Since fulllength p53 exists as a tetramer, a p53 tetramer will likely interact with two BCL-xL molecules. Our structure is different from the previous NMR model ${ }^{17}$. The difference may be due to the following factors: 1 , the size of $\mathrm{p} 53$-dimer/BCL-xL complex exceeds the detection limit of NMR Spectroscopy; 2, the interaction between p53 and BCL-xL may be highly dynamic in solution, and beyond the limit of NMR detection. Our structural studies reveal that p53 dimer can bind BCL-xL in a well-defined and specific manner. These observations together with our biochemical and cellular data, provide insights into the transcription-independent activity of p53-mediated mitochondrial apoptosis and the mechanism of its inactivation by p53 mutations.

\section{Methods}

Escherichia coli Rosetta BL21 (DE3). Escherichia coli cells Trans10 (Transgen, CD101-01) and BL21 (DE3) (Transgen, CD601-02) were cultured in LB medium at $37^{\circ} \mathrm{C}$. For protein expression, BL21 Rosetta (DE3) cells were grown in LB at $37^{\circ} \mathrm{C}$ until optical density at $600 \mathrm{~nm}$ reached 0.8 . Then isopropyl- $\beta$-D- thiogalactopyranoside was added to a final concentration of $0.2 \mathrm{mM}$, and the culture was transferred to a $25^{\circ} \mathrm{C}$ shaker for a further 12 hours.

H1299 cells. H1299 cells (American Type Culture Collection) were cultured in RPMI Medium 1640 basic (Gibco, C11875500BT) supplemented with 10\% fetal bovine serum (Gibco, 10091148), 1\% penicillin and streptomycin (Gibco, 10378016) under cell culture conditions $\left(37^{\circ} \mathrm{C}, 5 \% \mathrm{CO} 2\right)$.

Plasmids. Human BCL-xL lacking the disordered a1- $\alpha 2$ loop (amino acids 45-84) and the 22 C-terminal residues was cloned into pET-28a vector (Novagen, 698643 ) and pGEx-6P1 (GE healthcare, 28-9546-48), respectively (Supplementary

Table 2). Human BCL-2 (amino acids 1-205) was cloned into pET-28a vector with a C-terminal $6 \times$ HIS-tag. Human p53 containing the DBD (p53-DBD, amino acids 92-292) was cloned into vector pMAL-c5x. Human p53 containing the DBD and tetramerization domain (p53-DT, amino acids 92-360) was cloned into modified pET-28a ${ }^{42}$ with a N-terminal $6 \times$ His-tag. For crystallization, a fusion protein coding p53-DBD connected via a Glycine-rich linker (GGGGSLVPRGSGGGGS) to BCL$\mathrm{xL}$ was PCR amplified and cloned into pET-28a vector. Full-length p53 and BCL$\mathrm{xL}$ used in cellular assays were cloned into the retroviral vector pQCXIH (Clontech, 631516). Schematic representation of all constructs was shown in Supplementary Fig. 1.

All BCL-xL and p53 plasmids were performed according to the manufacturer's instructions for the ClonExpress II One Step Cloning Kit (Vazyme, C112). All mutants were constructed by PCR mutagenesis using the KOD Plus mutagenesis 
kit (TOYOBO, SMK-101) using the wild-type plasmid as the template. All plasmids were confirmed by DNA sequencing (Tsingke). Plasmids generated in this study have been deposited to NHC Key Laboratory of Cancer Proteomics of XiangYa Hospital.

Protein expression and purification. All proteins were expressed in Escherichia coli BL21 (DE3) cells and induced by $0.2 \mathrm{mM}$ isopropyl $\beta$-D-1thiogalactopyranoside (IPTG) for 12 hours at $18^{\circ} \mathrm{C}$. The fused BCL-xL-p53-DBD proteins were purified by nickel affinity chromatography (GE Healthcare, 17-531802) and cation-exchange chromatography (Mono S 5/50GL, GE Healthcare, 175168-01). Then the protein was further purified by size exclusion chromatography (Superdex 200 10/300 GL, GE Healthcare, 17-5175-01). Peak fractions were collected and concentrated to $10 \mathrm{mg} / \mathrm{ml}$ and stored at $-80^{\circ} \mathrm{C}$ until use.

His-tagged BCL-xL and BCL-2 proteins were purified by nickel affinity chromatography, followed by anion-exchange chromatography (Mono Q 5/50GL, GE Healthcare, 17-5166-01). GST-BCL-xL was purified by glutahtione sepharose (GE Healthcare, 17-0756-01). p53-DBD protein was firstly purified by Amylose chromatography (Amylose Resine, New England Biolabs, E8021L) followed by incubation with PreScission protease at $4^{\circ} \mathrm{C}$ to remove MBP-tag. p53-DT protein was firstly purified by nickel affinity chromatography and the N-terminal $6 \times$ Histag was removed by PreScission protease. Then the p53 proteins were further purified by cation-exchange chromatography (Mono S 5/50GL, GE Healthcare, 17-5168-01). All mutant proteins were purified by the same methods as wild-type proteins.

Crystallization and structure determination. Crystals of the BCL-xL-p53-DBD fusion protein were grown at $18{ }^{\circ} \mathrm{C}$ by the hanging-drop method with a reservoir buffer of $0.2 \mathrm{M}$ sodium malonate $\mathrm{pH} 7.0$ and $20 \%$ polyethylene glycol $3,350(\mathrm{w} / \mathrm{v})$ (Hampton Research, HR2-126). For data collection, a single crystal was soaked in well solution plus $20 \%$ glycerol (v/v) and flash-frozen in liquid nitrogen. Data were collected at the BL17U ${ }^{43}$ and BL19U1 ${ }^{44}$ beamlines of Shanghai Synchrotron Radiation Facility (SSRF). Then the data were processed using HKL2000 ${ }^{45}$. The structure was solved by molecular replacement with phaser from PHENIX package $^{46}$ using the previously solved p53 structure (PDB: 3KMD) ${ }^{4}$ and BCL-xL structure (PDB: $2 \mathrm{YXJ})^{22}$ as search templates. Then the structure was refined with phenix.refine and model building was performed using WinCoot ${ }^{47}$. Translationalliberation-screw (TLS) refinement was used during the last stages of refinement. Graphical representations of the structure were generated using PyMOL ${ }^{48}$. The statistics of the crystallographic analysis are presented in Supplementary Table 1.

Isothermal titration calorimetry (ITC). ITC measurements were performed over 25 injections of $2 \mu \mathrm{L}$ each at $25^{\circ} \mathrm{C}$ in $50 \mathrm{mM}$ Tris pH 7.0, $40 \mathrm{mM} \mathrm{NaCl}, 1 \mathrm{mM}$ TCEP, using a Nano ITCRun (TA Instruments). p53 protein $(100 \mu \mathrm{M})$ was titrated into solutions containing $10-30 \mu \mathrm{M}$ BCL-xL or BCL-2. p53 protein $(100 \mu \mathrm{M})$ titrated into buffer was used as a background. All titrations were performed for triplicate independent experiments. To remove any protein precipitate, the samples were centrifuged before the experiments. The titration data were analyzed using the NanoAnalyze software.

Crosslinking analysis. Purified p53-DT or p53-DBD proteins and BCL-xL were desalted to buffer containing $20 \mathrm{mM}$ HEPES pH 7.0, $100 \mathrm{mM} \mathrm{NaCl}, 1 \mathrm{mM}$ TCEP. Then $10 \mu \mathrm{L}$ p53-DBD or p53-DT protein at $20 \mu \mathrm{M}$ was mixed with $10 \mu \mathrm{L}$ PBS or $10 \mu \mathrm{L}$ BCL-xL protein at $20 \mu \mathrm{M}$ on ice for $30 \mathrm{~min}$. Then $2 \mu \mathrm{L} 1 \%$ glutaraldehyde (SIGMA, G5882) was added to the mixture and incubated for $15 \mathrm{~min}$ at $37^{\circ} \mathrm{C}$. Products of the cross-linking reactions were analyzed by 4-12\% SDS-PAGE (M00652, GenScript) as indicated and then silver stained (P0017S, Beyotime).

GST pull-down. $50 \mu$ L Purified GST tag or GST-BCL-xL at a concentration of $10 \mu \mathrm{M}$ was incubated with $50 \mu \mathrm{L}$ p53-DBD or p53-DT protein at $10 \mu \mathrm{M}$ for 1 hour at $4{ }^{\circ} \mathrm{C}$ in PBS buffer containing $1 \mathrm{mM}$ TCEP. The mixture was incubated with $20 \mu \mathrm{l}$ GST beads (GE Healthcare, 17-0756-01) for $10 \mathrm{~min}$ at $4{ }^{\circ} \mathrm{C}$. Then the GST beads were washed with PBS buffer containing $1 \mathrm{mM}$ TCEP and $0.5 \%$ Triton X100, resuspended with $100 \mu \mathrm{l}$ of protein loading buffer and loaded onto a $15 \%$ SDSPAGE gel. p53 proteins were detected by western blotting using anti-p53 antibody (abcam, ab26, GR3213177-1, a working concentration of $2 \mu \mathrm{g} / \mathrm{ml}$ ), and GST or GST-BCL-xL was detected by anti-GST tag antibody (Abbkine, 2A8, ATTJA0301, 1:5,000 working dilution).

Cell transfection. All plasmids were transfected with Lipofectamine 2000 (Invitrogen, 11668027) according to the manufacturer's protocol. The transfection efficiency was verified by western blotting.

Coimmunoprecipitation. $\mathrm{H} 1299$ cells were lysed in the precipitation assay buffer containing $0.3 \%$ Nonidet $\mathrm{P} 40,150 \mathrm{mM} \mathrm{NaCl}, 50 \mathrm{mM}$ Tris-HCl pH 7.5 and multiple protease inhibitors. Cell lysates were cleared with protein $\mathrm{G}$-agarose beads (Roche, 11719416001 ) for 1 hour at $4{ }^{\circ} \mathrm{C}$. Two micrograms of anti-p53 antibody (Cell Signaling Technology, 9282 S, 5), anti-Flag antibody (Cell Signaling Technology, 8146 $\mathrm{T}, 3,1: 1: 50$ working dilution) or normal IgG (Santa Cruz Biotechnology, sc-2748) were separately added to the cell lysates and incubated overnight at $4{ }^{\circ} \mathrm{C}$ on a rotator. Protein G beads $(30 \mu \mathrm{L})$ were added to the cell lysates and incubated for 3 hours. Beads were washed 3 times with precipitation assay buffer and boiled in RIPA lysis buffer (Beyotime, P0013B), then subjected to SDS-PAGE and analyzed by western blotting.

Cell-apoptosis analysis. H1299 cells $\left(1 \times 10^{5}\right)$ were seeded in 12 -well plates, cultured for 48 hours, and transiently transfected with $1 \mu \mathrm{g}$ of the indicated p53 plasmids. Then, the cells were treated with a Propidium Iodide (PI)/Fluorescein Isothiocyanate (FITC)-Annexin V Staining Kit (Vazyme, A211-01) and subjected to flow cytometry. A total of $10^{6}$ cells were analyzed for each sample, and each condition was tested in three independent samples. FlowJo software was utilized to calculate the apoptotic cell rate.

Western blotting analysis. Experiments were conducted using the following primary antibodies: anti-p53 antibody (1:1,000 working dilution), anti-BCL-xL antibody (Cell Signaling Technology, 2764 S, 9, 1:1,000 working dilution) and anticaspase 3 antibody (Proteintech, 19677-1-AP, 1:1,000 working dilution) as indicated. The secondary antibodies HRP-conjugated Goat Anti-Mouse IgG (Abbkine, A21010, ATSDE1601, 1:2,000 working dilution), HRP Goat Anti-Rabbit IgG (Absin, abs20040, AS004, 1:2,000 working dilution) were used. Bands were visualized by enhanced chemiluminescence detection reagents (Vazyme, E411-04). Bands of western blotting were quantified using ImageJ software.

Quantification and statistical analysis. GraphPad Prism 7.0 was used to perform one-way ANOVA followed by Dunnett's multiple comparisons test using wild-type group as a control to determine $P$-values. Data are presented as mean. Error bars indicate standard error of the mean (SEM) $\left(n=3,{ }^{* *} P<0.01,{ }^{* *} P<0.001\right)$.

Reporting summary. Further information on research design is available in the Nature Research Reporting Summary linked to this article.

\section{Data availability}

The structure models used in the study from the Protein Data Bank under the following accession codes: $3 \mathrm{KMD}, 2 \mathrm{YXJ}, 4 \mathrm{QVE}, 4 \mathrm{PPI}, 2 \mathrm{XA} 0,2 \mathrm{MEJ}, 2 \mathrm{H} 1 \mathrm{~L}, 1 \mathrm{KZY}, 4 \mathrm{HJE}, 4 \mathrm{XR} 8$, $1 Y C S$. The coordinates and structure factors are deposited in the Protein Data Bank under the accession codes 6LHD (p53/BCL-xL complex). All other relevant data supporting the key findings of this study are available within the article and its Supplementary Information files or Source data. Source data are provided with this paper.

Received: 30 September 2020; Accepted: 24 March 2021; Published online: 16 April 2021

\section{References}

1. Levine, A. J. p53: 800 million years of evolution and 40 years of discovery. Nat. Rev. Cancer 20, 471-480 (2020).

2. Kastenhuber, E. R. \& Lowe, S. W. Putting p53 in context. Cell 170, 1062-1078 (2017).

3. Joerger, A. C. \& Fersht, A. R. The p53 pathway: origins, inactivation in cancer, and emerging therapeutic approaches. Annu. Rev. Biochem. 85, 375-404 (2016).

4. Chen, Y., Dey, R. \& Chen, L. Crystal structure of the p53 core domain bound to a full consensus site as a self-assembled tetramer. Structure 18, 246-256 (2010).

5. Chen, Y. et al. Structure of $\mathrm{p} 53$ binding to the BAX response element reveals DNA unwinding and compression to accommodate base-pair insertion. Nucleic Acids Res. 41, 8368-8376 (2013).

6. Kitayner, M. et al. Diversity in DNA recognition by p53 revealed by crystal structures with Hoogsteen base pairs. Nat. Struct. Mol. Biol. 17, 423-429 (2010)

7. Green, D. R. \& Kroemer, G. Cytoplasmic functions of the tumour suppressor p53. Nature 458, 1127-1130 (2009).

8. Hafner, A., Bulyk, M. L., Jambhekar, A. \& Lahav, G. The multiple mechanisms that regulate p53 activity and cell fate. Nat. Rev. Mol. Cell Biol. 20, 199-210 (2019).

9. Chipuk, J. E. et al. Direct activation of Bax by p53 mediates mitochondrial membrane permeabilization and apoptosis. Science 303, 1010-1014 (2004).

10. Leu, J. I., Dumont, P., Hafey, M., Murphy, M. E. \& George, D. L. Mitochondrial p53 activates Bak and causes disruption of a Bak-Mcl1 complex. Nat. Cell Biol. 6, 443-450 (2004).

11. Mihara, M. et al. p53 has a direct apoptogenic role at the mitochondria. Mol. Cell 11, 577-590 (2003). 
12. Singh, R., Letai, A. \& Sarosiek, K. Regulation of apoptosis in health and disease: the balancing act of BCL-2 family proteins. Nat. Rev. Mol. Cell Biol. 20, 175-193 (2019)

13. Li, M., Wang, D., He, J., Chen, L. \& Li, H. Bcl-XL: a multifunctional antiapoptotic protein. Pharm. Res. 151, 104547 (2020).

14. Ngoi, N. Y. L. et al. Targeting mitochondrial apoptosis to overcome treatment resistance in cancer. Cancers 12, https://doi.org/10.3390/cancers12030574 (2020).

15. Lessene, G., Czabotar, P. E. \& Colman, P. M. BCL-2 family antagonists for cancer therapy. Nat. Rev. Drug Disco. 7, 989-1000 (2008).

16. Petros, A. M., Gunasekera, A., Xu, N., Olejniczak, E. T. \& Fesik, S. W. Defining the p53 DNA-binding domain/Bcl-x(L)-binding interface using NMR. FEBS Lett. 559, 171-174 (2004).

17. Follis, A. V. et al. The DNA-binding domain mediates both nuclear and cytosolic functions of p53. Nat. Struct. Mol. Biol. 21, 535-543 (2014).

18. Sot, B., Freund, S. M. \& Fersht, A. R. Comparative biophysical characterization of p53 with the pro-apoptotic BAK and the anti-apoptotic BCL-xL. J. Biol. Chem. 282, 29193-29200 (2007).

19. Hagn, F. et al. BclxL changes conformation upon binding to wild-type but not mutant p53 DNA binding domain. J. Biol. Chem. 285, 3439-3450 (2010).

20. Tomita, Y. et al. WT p53, but not tumor-derived mutants, bind to Bcl2 via the DNA binding domain and induce mitochondrial permeabilization. J. Biol. Chem. 281, 8600-8606 (2006).

21. Dominguez, C., Boelens, R. \& Bonvin, A. M. HADDOCK: a protein-protein docking approach based on biochemical or biophysical information. J. Am. Chem. Soc. 125, 1731-1737 (2003).

22. Lee, E. F. et al. Crystal structure of ABT-737 complexed with Bcl-xL: implications for selectivity of antagonists of the Bcl-2 family. Cell Death Differ. 14, 1711-1713 (2007).

23. Muchmore, S. W. et al. X-ray and NMR structure of human Bcl-xL, an inhibitor of programmed cell death. Nature 381, 335-341 (1996).

24. Reddy Chichili, V. P., Kumar, V. \& Sivaraman, J. Linkers in the structural biology of protein-protein interactions. Protein Sci. 22, 153-167 (2013).

25. Friedman, P. N., Chen, X., Bargonetti, J. \& Prives, C. The p53 protein is an unusually shaped tetramer that binds directly to DNA. Proc. Natl Acad. Sci. USA 90, 3319-3323 (1993).

26. Wang, Y., Rosengarth, A. \& Luecke, H. Structure of the human p53 core domain in the absence of DNA. Acta Crystallogr D. Biol. Crystallogr 63, 276-281 (2007).

27. Tidow, H. et al. Quaternary structures of tumor suppressor p53 and a specific p53 DNA complex. Proc. Natl Acad. Sci. USA 104, 12324-12329 (2007).

28. Cho, Y., Gorina, S., Jeffrey, P. D. \& Pavletich, N. P. Crystal structure of a p53 tumor suppressor-DNA complex: understanding tumorigenic mutations. Science 265, 346-355 (1994).

29. Lee, E. F. \& Fairlie, W. D. The structural biology of Bcl-xL. Int. J. Mol. Sci. 20, https://doi.org/10.3390/ijms20092234 (2019).

30. Rajan, S., Choi, M., Baek, K. \& Yoon, H. S. Bh3 induced conformational changes in $\mathrm{Bcl}-\mathrm{Xl}$ revealed by crystal structure and comparative analysis. Proteins 83, 1262-1272 (2015).

31. O’Neill, J. W., Manion, M. K., Maguire, B. \& Hockenbery, D. M. BCL-XL dimerization by three-dimensional domain swapping. J. Mol. Biol. 356 , 367-381 (2006).

32. Rajan, S. et al. Structural transition in $\mathrm{Bcl}-\mathrm{xL}$ and its potential association with mitochondrial calcium ion transport. Sci. Rep. 5, 10609 (2015).

33. Ku, B., Liang, C., Jung, J. U. \& Oh, B. H. Evidence that inhibition of BAX activation by BCL-2 involves its tight and preferential interaction with the BH3 domain of BAX. Cell Res. 21, 627-641 (2011).

34. Burke, P. J. Mitochondria, bioenergetics and apoptosis in cancer. Trends Cancer 3, 857-870 (2017).

35. Mohammad, R. M. et al. Broad targeting of resistance to apoptosis in cancer. Semin Cancer Biol. 35(Suppl), S78-S103 (2015).

36. Fridman, J. S. \& Lowe, S. W. Control of apoptosis by p53. Oncogene 22, 9030-9040 (2003).

37. Martinez-Zapien, D. et al. Structure of the E6/E6AP/p53 complex required for HPV-mediated degradation of p53. Nature 529, 541-545 (2016).

38. Lilyestrom, W., Klein, M. G., Zhang, R., Joachimiak, A. \& Chen, X. S. Crystal structure of SV40 large T-antigen bound to p53: interplay between a viral oncoprotein and a cellular tumor suppressor. Genes Dev. 20, 2373-2382 (2006).

39. Joo, W. S. et al. Structure of the 53BP1 BRCT region bound to $\mathrm{p} 53$ and its comparison to the Brcal BRCT structure. Genes Dev. 16, 583-593 (2002).
40. Gorina, S. \& Pavletich, N. P. Structure of the p53 tumor suppressor bound to the ankyrin and SH3 domains of 53BP2. Science 274, 1001-1005 (1996)

41. Rivlin, N., Brosh, R., Oren, M. \& Rotter, V. Mutations in the p53 tumor suppressor gene: important milestones at the various steps of tumorigenesis. Genes Cancer 2, 466-474 (2011).

42. Chen, X. et al. Structural basis for DNA recognition by FOXC2. Nucleic Acids Res. 47, 3752-3764 (2019).

43. Wang, Q.-S. et al. Upgrade of macromolecular crystallography beamline BL17U1 at SSRF. Nucl. Sci. Tech. 29, 68 (2018).

44. Zhang, W.-Z. et al. The protein complex crystallography beamline (BL19U1) at the Shanghai synchrotron radiation facility. Nucl. Sci. Tech. 30, 170 (2019).

45. Otwinowski, Z. \& Minor, W. in Methods in Enzymology Vol. 276 307-326 (Academic Press, 1997).

46. Adams, P. D. et al. The Phenix software for automated determination of macromolecular structures. Methods 55, 94-106 (2011).

47. Emsley, P. \& Cowtan, K. Coot: model-building tools for molecular graphics. Acta Crystallogr D. Biol. Crystallogr 60, 2126-2132 (2004).

48. Warren, L. D. PyMOL User's Guide. Delano Scientific, San Carlos (2004).

\section{Acknowledgements}

This work was supported by the National Natural Science Foundation of China (grants 81570537 and 81974074 to Y.C.; grant 31900880 to H.W., grant 81902858 to Y.Z.), China Postdoctoral Science Foundation (2019M652805), Science and Technology Planning Project of Hunan Province (2018TP1017). We thank the staffs from BL17U/BL19U1 beamline of National Facility for Protein Science in Shanghai (NFPS) at Shanghai Synchrotron Radiation Facility, for assistance during data collection. We thank Dr. Michael R. Stallcup for proofreading.

\section{Author contributions}

H.W., Y.Z., L.Q., Y.F., Y.L., H.W., L.J. and X.C. performed experiments; H.W., S.D., M.G. and J.L. performed data collection and structure determination. H.W., Y.Z., Z.C., L.C. and Y.C. analyzed the data. H.W., Y.Z. and Y.C. prepared the manuscript.

\section{Competing interests}

The authors declare no competing interests.

\section{Additional information}

Supplementary information The online version contains supplementary material available at https://doi.org/10.1038/s41467-021-22655-6.

Correspondence and requests for materials should be addressed to Y.Z. or Y.C.

Peer review information Nature Communications thanks Carol Lim, Marc Kvansakul, and other, anonymous, reviewer(s) for their contributions to the peer review of this work. Pee review reports are available.

Reprints and permission information is available at http://www.nature.com/reprints

Publisher's note Springer Nature remains neutral with regard to jurisdictional claims in published maps and institutional affiliations.

Open Access This article is licensed under a Creative Commons Attribution 4.0 International License, which permits use, sharing, adaptation, distribution and reproduction in any medium or format, as long as you give appropriate credit to the original author(s) and the source, provide a link to the Creative Commons license, and indicate if changes were made. The images or other third party material in this article are included in the article's Creative Commons license, unless indicated otherwise in a credit line to the material. If material is not included in the article's Creative Commons license and your intended use is not permitted by statutory regulation or exceeds the permitted use, you will need to obtain permission directly from the copyright holder. To view a copy of this license, visit http://creativecommons.org/ licenses/by/4.0/.

(C) The Author(s) 2021 